\section{A MICROSCOPE AS THE SMALLEST PEN}

Stephen W. Carmichael, ${ }^{1}$ Mayo Clinic

In a movie I saw recently, a young Will Shakespeare was seen to repeatedly dip the nib of a quill into a reservoir of ink and scrawl on pieces of paper until, finally, what we know as "Romeo and Juliet" was penned. As pointed out by Richard Piner, Jin Zhu, Feng Xu, Seunghun Hong, and Chad Mirkin, ${ }^{2}$ this technology is much older than Shakespeare, dating back about 4000 years. But even technology this old can change.

As you are well aware, making devices on a smaller and smaller scale (nanofabrication) is certain to change our future way of life. Nanofabrication frequently relies on lithographic methods where a pattern is superimposed on a resistive film and the film is chemically etched to create a structure that conforms to the pattern. Piner et al. have merged modern lithographic methods with the technology used by Shakespeare, among others, where a sharp object is used to transfer a visible fluid by capillary forces to a suitable substrate, such as paper. They call this new method "dip-pen" nanolithography.

In simplistic terms, one would consider the sharpness of the "nib," the roughness of the "paper," and the molecular composition of the "ink" to be the limiting factors to drawing the finest line possible. For their nib. Piner et al. used the tip of an atomic force microscope (AFM). This is only a few atoms in diameter. However, when used in air, an aqueous meniscus fills the capillary formed between the tip and surface, a physical phenomenon that has plagued atomic force microscopy since it was invented. Depending on the relative humidity and the wetting properties of the substrate, the size of the meniscus that forms in the narrow gap between the tip and substrate can vary.

For the substrate (the "paper"), Piner et al. used a gold film annealed to mica. It turned out that the roughness of the gold was the factor that limited the width of line that could be drawn. They could make a line $30 \mathrm{~nm}$ wide, the average diameter of a grain of gold. Theoretically, narrower lines could be drawn on a smoother surface.

The "ink" they used was 1-octadecanethiol (ODT). When choosing the ink, an important characteristic is that it can be read, and this alkanethiol can be easily imaged from the unmodified gold substrate by lateral force microscopy. ODT is a moderately air-stable molecule that apparently flows from the tip of the AFM to the gold substrate by capillary action, just like ink from the nib of a pen. Unlike conventional inks, the ODT reacts with the surface as the nanoline is formed, resulting a stable molecule-based nanostructure. For their experiments, Piner et al. dipped a silicon nitride tip in a solution of ODT, dried the tip, then scanned a gold substrate in a raster pattern. Dark lines stood out from the background. Measurements were consistent with the lines being formed by the ODT, and not water or the solvent used to dissolve the ODT.

Scan speed and relative humidity were other factors that influenced the resolution of their system. A slower scan speed (longer tip-substrate contact time) gave a relatively broader line. A lower relative humidity gave a narrower line. These two factors appear to be inversely related to each other.

Piner et al. point out that the ultimate resolution of dip-pen nanolithography has not been reached, in fact, they're not sure what the limit is. However, it is clear that they have developed a relatively simple but powerful method for moving molecules from an AFM tip to a substrate in a well-controlled manner. They didn't set out to write "Romeo and Juliet" on the head of a pin, but they have shown that this is possible! But seriously, this technique will be particularly useful for making nanoscale devices functional, and this accelerates our move to the future!

1. The author gratefully acknowledges'Dr. Chad Mirkin for reviewing this article.

2. Piner, R.D., J. Zhu, F. Xu, S. Hong, and C.A. Mirkin, "Dip-Pen" nanolithography, Science 283:661-663, 1999.

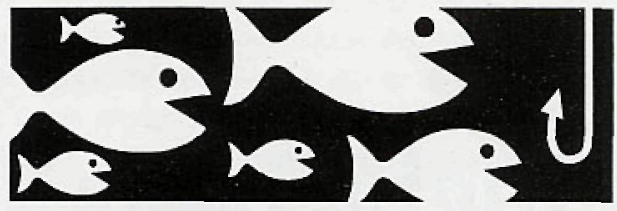

\title{
Front Cover Image
}

\section{Anaglyph Images of Pollen from Garden Plants (700X)}

The larger pollen grains are from Asiatic Lily while the smaller ones are undentified. Stereo images were taken on a JEOL JSM-840 SEM at $5 \mathrm{kV}$ and 7 degrees tilt. Digital files were captured at a resolution of $1280 \times 960$ with a 160 second dwell time. The image was manipulated as indicated in the article "Making Anaglyph Images from SEM Images Using Adobe Photoshop" by Debby Sherman (Dept. of Botany \& Plant Pathology, Purdue University) from the January 1999 issue of this publication.

\section{mICROSCOPY TODAY}

The publication is mailed, ten times a year, at no charge to some 8,400 microscopists in the United States, and some 500 overseas - all of which have requested subscriptions. White the publication is sent at no charge in the U.S., we regret that we must charge for international subscriptions.

Canada, Mexico; Latin and South America: $\$ 40$ for one year, $\$ 70$ for two years

Other International: $\$ 55$ for one year, $\$ 95$ for two years

Payment may be made by check or credit card.

\section{mICROSCOPY TOPAY}

PO Box 620122, Middleton, WI 53562

Tel.: (608)836-1970 • Fax: (608)836-1969 • eMail: MicroToday@aol.com•http://www.microscopy-today.com Copyright in 1999 by Today Enterprises. All rights reserved 

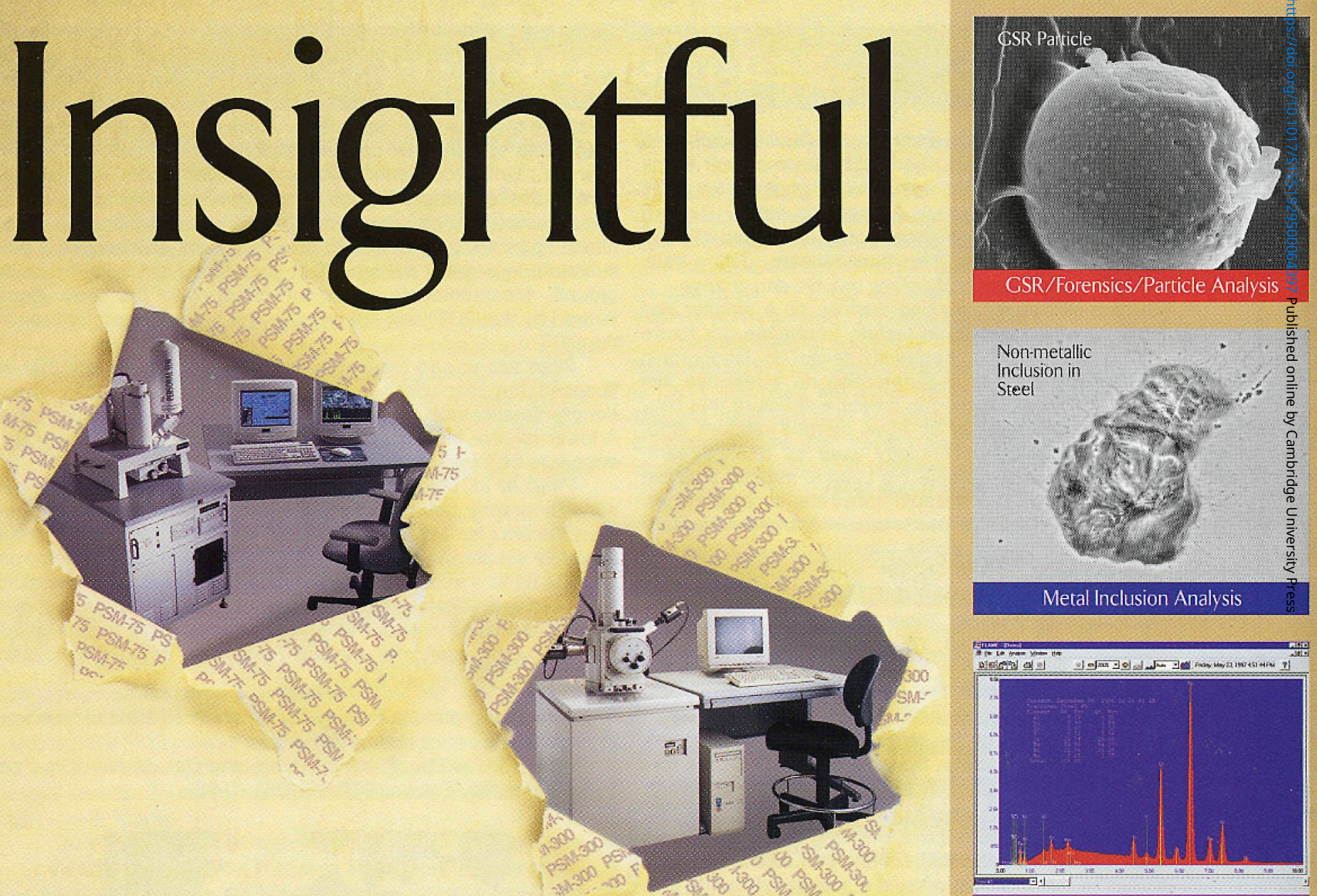

\section{The Right Tools for a Broad Spectrum of Applications}

Better insights, better analysis

Looking for better, more insightful analysis? Look no further. The PSM-300 and the PSM-75 are simple-to-use analytical instruments that give you multiple, valuable insights related to your specific application. Better insights for a more comprehensive analysis. Better insights for critical information. Better insights to make the right decisions.

Better insights for more effective solutions. Better insights to cost-saving solutions. And the list of insights goes on and on.

Customized for your specific application

The PSM- 300 or the PSM-75 can be easily customized to your specific application, some of which include:
- Automated Gun Shot Residue Analysis

- Automated Inclusion Analysis

- Automated Wear Debris Analysis

- Automated Particle Analysis

- Automated Fiber Analysis

To get more insights into easy, more effective microanalysis at a truly affordable price call/4-800-538-6850.
Metal Inclusion Analysis

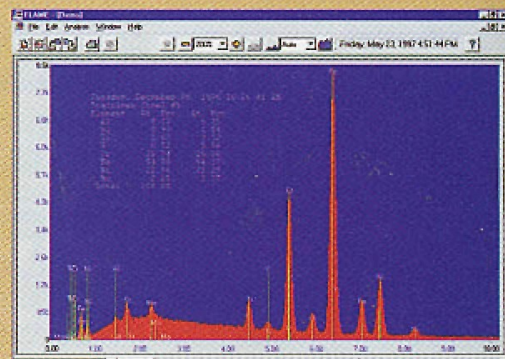

Inclusion EDX Spectrum
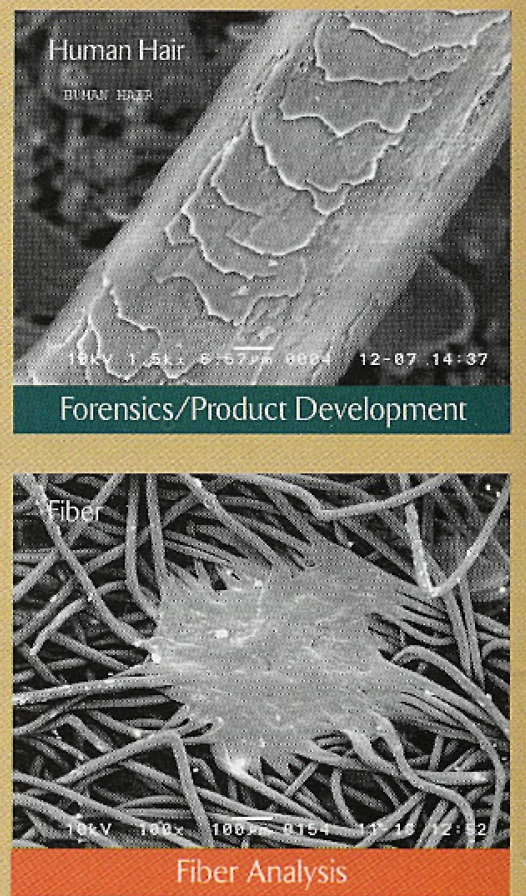